\title{
La Province De l'Ituri En République Démocratique Du Congo Face Aux Cycles Des Violences En Répétition. Essai D'une Analyse Comparative Des Dynamiques De 1999 À 2003 Et De 2017 À 2019
}

\section{Henri Mbuna Dhedonga,}

Diplômé d'Etudes Supérieures en Science Politique de l'Université de Kinshasa et Chef de Travaux à l'Université de Bunia. Ses Recherches Couvrent les Domaines de la Sociologie Politique et plus particulièrement la Transmutation des Partis Politiques en Groupes Armés

\section{Touré Maliaka Sefu,}

Candidat au Diplôme d'Etudes Supérieures (DES) en Science Politique à l'Université de Kisangani. Ses Domaines de Recherches sont principalement orientés vers la Sociologie des Conflits, la Sociologie Politique et la Sociologie Électorale

Justin Bahinduka Besisa,

Prépare un Diplôme d'Etudes Supérieures en Relations internationales de 1'Université de Kisangani en RDC depuis 2016. Ses Recherches Couvrent les Domaines de la Politique Étrangère, de la Géopolitique et des Théories de la Coopération Internationale. Il se Spécialise Actuellement sur la Coopération Entre la RDC et l'Ouganda dont le Jalon a été jeté en 2007 par un Accord

Signé à Ngurdoto en Tanzanie

Prince Lonu Lotsima, Assistant au Centre de Recherche en Langues et Cultures Africaines/Kisangani et à l'Université de Bunia en République Démocratique du Congo

Doi:10.19044/esj.2019.v15n35p105 URL:http://dx.doi.org/10.19044/esj.2019.v15n35p105

\section{Résumé}

Décrite comme une zone ensanglantée de conflit, la province de l'Ituri s'est immergée entre 1998 et 2017, avec des interruptions sporadiques, dans une guerre ethno-tribale et dans un embrasement d'agressions de la RDC par ses voisins (Rwanda et Ouganda), ayant joué aux sapeurs-pompiers pyromanes pour satisfaire leur boulimie des ressources naturelles de cette partie de la République Démocratique du Congo. La pression internationale sur ces pays agresseurs de la RDC pour leur retrait immédiat et la décision d'expédier sans condition une Force Multinationale Intérimaire d'Urgence 
(FMIU) dont la France fut la première à organiser « l'opération Artémis » en 2002, ont mis en déroute les auteurs des crimes contre l'humanité observés dans la province de l'Ituri. Ainsi le pays recouvra son intégrité territoriale et le processus démocratique s'acheva à l'instauration effective de la troisième république en RDC avec les élections de 2006. Par des formules fortuites, des événements similaires se dupliquent en 2017 et 2019 avec une cadence accélérée que jadis. Un tableau sombre d'insécurité se dresse encore dans la province de l'Ituri, justifié par des hargneux accrochages entre les Forces Armées de la République Démocratique du Congo (FARDC) et les hors la loi qualifiés par le pouvoir public des « assaillants » sans identité au départ, puis identifiés par l'armée nationale à la milice de CODECO d'un sujet Lendu nommé Ngudjolo, comprenant en majorité certains jeunes égarés de la communauté Bbale (Lendu), et qui massacre plusieurs tribus dont principalement le Hema dans le territoire de Djugu et les Alur à Mahagi. Depuis lors, deux ethnies (HEMA et LENDU) font l'histoire belliqueuse du Territoire de DJUGU et plusieurs analystes s'accordent sur l'origine foncière du conflit qui les oppose. S'inscrivant dans la logique de leur dépassement, cette note d'analyse tente d'établir les écarts et les rapprochements de ce conflit à ces deux épisodes évoqués ci-dessus.

Mots-clés : Guerres Interethniques, Ituri, Épisodes, Analogies, Altérités 


\title{
Interethnic Wars in Ituri in Two Episodes: A Study on its Analogies and its Differences
}

\section{Henri Mbuna Dhedonga,}

Diplômé d'Etudes Supérieures en Science Politique de l'Université de

Kinshasa et Chef de Travaux à l'Université de Bunia. Ses Recherches Couvrent les Domaines de la Sociologie Politique et plus particulièrement la Transmutation des Partis Politiques en Groupes Armés

Touré Maliaka Sefu,

Candidat au Diplôme d'Etudes Supérieures (DES) en Science Politique à l'Université de Kisangani. Ses Domaines de Recherches sont principalement orientés vers la Sociologie des Conflits, la Sociologie Politique et la

Sociologie Électorale

\section{Justin Bahinduka Besisa,}

Prépare un Diplôme d'Etudes Supérieures en Relations internationales de l'Université de Kisangani en RDC depuis 2016. Ses Recherches Couvrent les Domaines de la Politique Étrangère, de la Géopolitique et des Théories de la Coopération Internationale. Il se Spécialise Actuellement sur la Coopération

Entre la RDC et l'Ouganda dont le Jalon a été jeté en 2007 par un Accord

Signé à Ngurdoto en Tanzanie

Prince Lonu Lotsima,

Assistant au Centre de Recherche en Langues et Cultures

Africaines/Kisangani et à l'Université de Bunia en

République Démocratique du Congo

\begin{abstract}
Described as a bloody zone of conflict between 1998 and 2017, the province of the Ituri experienced sporadic interruptions in an ethno-tribal war and in kindling of aggressions of the DRC by its neighbors (Rwanda and Uganda). These external actors played the role of pyromaniac firemen in this war of the Ituri to satisfy their bulimia of the natural resources of this part of the Republic. The international pressure on the aggressor countries of the DRC based on their immediate withdrawal and the decision to dispatch without condition the Multinational Interim Emergency Force (MIEF), of which France was the first to organize the "Artémis operation", routed the perpetrators of the crimes against humanity observed in the province of the Ituri. As a result, the country regained its territorial integrity and the democratic process ended up at the institution of the third republic in DRC
\end{abstract}


with the elections of 2006. By fortuitous formulas, similar events duplicated themselves in 2017 and 2019 with a more accelerated cadence than previously. A gloomy picture of insecurity still rises in the province of Ituri, justified by snarling clashes between the Armed Forces of the Democratic Republic of Congo (FARDC) and the outlaws qualified by the public authorities of the "attackers" without identity initially. After then, it was identified by the national army to the CODECO militia of a Lendu subject named Ngudjolo. This comprises of some misguided young people from the Lendu community, and those who massacred several tribes including mainly Hema in the territory of Djugu and the Alur in Mahagi. Ever since then, two ethnic groups (HEMA and LENDU) have made the warlike history of the territory of DJUGU. Also, several analysts agree on the fundamental origin of the conflict that opposes them. Enrolling in the logic of their overtaking, this analytical paper focuses on establishing the gap and the correspondence of this conflict in these two above mentioned episodes.

Keywords: Interethnic Wars, Ituri, Episodes, Analogies, Differences

\section{Introduction}

Du district en province, l'Ituri se calfeutre toujours dans une érosion de la cohabitation ethnique dans le territoire de Djugu, habité principalement par les Lendu et les Hema, deux ethnies en conflit répétitif.

L'évolution du statut de l'Ituri n'a pas boosté l'amélioration des conditions sécuritaires en Ituri, une province qui continue à sombrer dans une spirale de violences infernales. Ainsi, l'Ituri reste l'otage des réseaux extrêmement violents que l'Etat peine à contenir.

Dans cette réflexion, les analogies et les altérités des deux guerres connues en Ituri sont dégagées en termes d'acteurs, des stratégies, des enjeux, des motivations ainsi que des ententes dudit conflit. Cela étant, la réflexion de cette note d'étude est guidée par la méthode géopolitique de François Thual, appuyée par une analyse comparative. Les techniques d'observation directe désengagée et celle documentaire ont, par ailleurs, fourni des informations nécessaires.

La première s'est focalisée sur le vécu quotidien de certains indicateurs possibles des guerres sans une intégration active des chercheurs. Elle a donc invité les chercheurs à regarder - avec une attention toute méritée- les faits tels qu'ils se reproduisent pour les étudier, les comprendre et les analyser. La seconde s'est penchée à l'analyse de la documentation disponible. Cette documentation a été regroupée selon qu'il s'agit des textes de rapports rendus publics par des structures intervenant directement/indirectement aux conflits d'une part, et, à des publications scientifiques d'autre part. 


\section{Genèse du conflit}

L'analyse de la genèse des guerres interethniques en Ituri invite à parler de deux peuples en présence, vivant dans les territoires de Djugu et Irumu. Il s'agit du peuple Bbale (Lendu) d'une part et du peuple Hema d'autre part.

En effet, les dynamiques migratoires qui ont conduit au peuplement de l'Ituri révèlent que le peuple Bale (Lendu) venu du Soudan au XVII ${ }^{\mathrm{e}}$ siècle, à la côte frontale de l'Ethiopie, a pénétré le pays qu'on appelle actuellement la RDC par le nord de l'Ituri, précisément par l'actuel territoire de Mahagi où ils ont trouvé et repoussé les Nyali (Bantu) vers l'ouest. Ayant conquis cet espace, ils y pratiquèrent l'agriculture. Par contre, les Hema, 'nilotique' et peuple agropastoral, auraient émigré en Ituri vers la fin du XVIII ${ }^{\mathrm{e}}$ siècle, via l'Ouganda, en provenance des hauts- plateaux du Sud-est (S. KAPUT0, 1982, pp. 35 et 38$)$.

Il est question dans ce point d'interroger l'histoire de ces deux peuples en présence afin de comprendre la dynamique des conflits qui les séparent au fil des années. Cela étant, cette note d'analyse replonge dans des raisons lointaines d'une part et celles récentes de l'autre part.

\section{Les raisons lointaines : de la ruse métropolitaine à un problème psychologique issu de l'accès aux services sociaux}

Le pouvoir coloniale belge n'a pas été exercé de façon à réunir les communautés congolaises de base : il sied de souligner que la stratégie de l'administration coloniale consistait à favoriser certains groupes au détriment d'autres, en inculquant des mythes d'infériorité et de supériorité pour mieux diviser et régner par la suite. Ainsi, après l'annexion de l'État Indépendant du Congo (EIC) au Congo Belge, en 1908, la gestion de la colonie fut confiée à trois pouvoirs qui s'équilibraient : l'administration, les missions catholiques et les grandes sociétés privées (Bernard L., 2014, p. 32-34).

Les Hema, campés en Ouganda et dans le souci d'atteindre l'Ituri, auraient profité du passage de l'explorateur anglais Henry Morton Stanley dans la région de l'Ituri pour faire route ensemble. De ce fait, ils auraient tiré une large sympathie et des dividendes. De leur côté, les Lendu déjà installés en Territoire d'Irumu notamment, empêchèrent Stanley de poursuivre son expédition aux environs de Bogoro, ce qui déterminera la conduite des colons par rapport aux Hema, qu'il percevra comme un peuple docile, et les Lendu, comme un peuple révolté.

Perpétuant cette séparation débutante, le pouvoir colonial favorisa l'accès libre du peuple Hema à l'instruction et généra une sorte de condamnation du peuple Lendu à l'exécution des travaux lourds. C'est dans cette logique qu'est né ce que nous appelons un problème psychologique issu de l'accès aux services sociaux. Ce problème s'explique par le complexe 
d'infériorité et/ou de supériorité qui trouve des explications chez les psychologues.

En effet, les psychologues s'accordent sur le fait que chaque individu naît avec plusieurs besoins et désirs qui lui conviennent et qu'il veut atteindre. Cependant, ces besoins et désirs semblent difficilement concevables pour un nombre de raisons, et c'est ce qui favorise le développement d'un sentiment d'infériorité (Saif, 2009). Par conséquent, les individus ayant un risque de développer ce type de complexe veulent vivre l'isolement en mettant en leur avant garde des signes de faible estime de soi qui puissent découler de leur appartenance ethnique, ce qui les exposent à des symptômes de dépression.

D’ores et déjà, Dan Fahey démontra que c'est en 1911 que le conflit éclata entre ces deux peuples, après l'assassinat en Territoire d'Irumu du grand chef Hema, Bomera par les Walendu Bindi (Fahey D., 2014, p.48).

Les hostilités s'étendraient dans le territoire de Djugu, où - en puisant de l'ouvrage de Nicaise Kibel'bel Okasur Les conflits armés en Ituri - il est reconnu qu'à l'ouverture des premières écoles à Fataki en 1918 et à Bambu en 1930, deux localités Lendu, ce sont les Hama Nord qui ont dû bénéficier de cet avantage au détriment des Lendu. Cette séparation trouve son fondement dans les fameuses recommandations consignées dans le carnet de voyage de Stanley parlant de l'incapacité du peuple Bbale (Lendu) à diriger et de sa démotivation au savoir scolaire mais prompts à tous travaux lourds. Les colons rendront par conséquent plus difficiles les conditions d'accès des jeunes Bbale à la scolarisation entre 1935 et 1940 (OKA N., 2016).

En 1940, et c'est ce qui a complètement contribué à distancier ces deux peuples, c'est le fait de l'annexion par l'autorité coloniale belge des villages Lendu de Lokpa et de Nombe dans la chefferie de Banyangu de Hema(Par la décision de l'autorité coloniale $\mathrm{n}^{\circ} 227$ du 27 Novembre 1940).

Après l'indépendance, cette série d'annexions s'est accélérée surtout dans des villages Lendu de la plaine de Semliki au secteur des Hema sud par l'autorité de la province de Kibali-Ituri en 1966.

En outre, les chercheurs de Human Wright Watch (HRW, 2003) révèlent que le régime colonial belge a accentué les divisions ethniques entre les deux communautés en essayant de réorganiser les chefferies traditionnelles en des groupes plus homogènes et en favorisant les Hema aux dépens des Lendu. Même après l'indépendance de 1960, les Hema sont restés dans leur position d'élite administrative, possédant terres et affaires : lors par exemple de la création du territoire de Kibali-Ituri en 1962, aucun Lendu n'a obtenu de positions importantes dans l'administration. Ce fait aura des sérieux et périlleux rebondissements en 1979, 1981et 1992 (OKA N., 2016). Jusqu'à ce jour, la contestation des limites entre le secteur des Bahema sud et la chefferie des Walendu Bindi constitue un des éléments essentiels du conflit Hema Lendu dans le territoire d'Irumu. 
En fin, il sied de révéler comme démontré ci-haut que bien que l'autorité coloniale eut semé des divisions entre les deux communautés, les autorités congolaises, après l'indépendance, ont de même appuyé ces divisions parce qu'en fait l'administration était acquise au plus docile, c'est-à-dire au Hema pour qui le pouvoir économique inspirait la force dans la conquête de nouvelles terres de pâturage.

2. Les raisons récentes d'une guerre interethnique de 1999 à 2004 :d'un problème de convoitise ordinaire des acteurs externes, à une bataille économique de la région.

En tout état de cause, nous remontons la résurgence des violences en Ituri au milieu des années 90, qui connut l'érosion d'un régime autoritaire de longue date et de l'avènement de l'AFDL (Alliance des forces démocratiques pour la libération du Congo) avec Laurent Désiré KABILA, le tombeur de Mobutu.

En effet, en octobre 1996, surgit à Fizi LD KABILA, à la tête d'une rébellion dite «guerre de libération » menée par l'AFDL, fortement financée et appuyée par les Rwandais, Burundais et Ougandais, avec comme objectif de renverser le régime de Mobutu. Le trio réussit les coups lorsqu'après plus de six mois de durs acharnements, les forces de l'AFDL pénétrèrent et prirent la ville de Kinshasa un certain 17 mai 1997.

Six mois après son accession au pouvoir, la lune de miel tourne aux divorces : non seulement que Laurent Désiré KABILA s'afficha hostile à la demande des Nations Unies de venir enquêter sur de massacres et autres violations graves commis par les génocidaires à l'Est du pays, particulièrement dans le Nord et sud Kivu, mais de surcroit il commença à accuser ceux qui l'avaient porté au pouvoir de piller, sans vergogne, les richesses du pays et de porter par ce fait atteinte à l'intégrité territoriale du pays (Touré, 2019).

En réponse à ce comportement « avilissant », surgit le 02 août 1998 une nouvelle rébellion, celle du Rassemblement Congolais pour la Démocratie (RCD) animée de prime à bord par Wamba dia Wamba, soutenue par le Rwanda, le Burundi et l'Ouganda. Au plus fort de la guerre ainsi déclenchée, le conflit Hema-Lendu éclate en Ituri en juin 1999.

Colette Braeckman insiste sur le fait que cette deuxième guerre du Congo a entraîné de surprenants renversements d'alliance et a démontré la fragilité des différents acteurs étatiques, tous englués dans des troubles intérieurs et des difficultés économiques (Braeckman, 1999).

En l'espace de quelques mois, après l'échec du coup d'Etat contre LD KABILA au courant de cette même année, c'est-à-dire 1998, la RDC, unie jusqu'alors sur toute l'étendue du territoire, s'est trouvée divisée en quatre zones distinctes à savoir: la zone sous autorité de LD KABILA basée à 
Kinshasa, la zone sous administration du Rassemblement Congolais pour la Démocratie (RCD) à Goma, la zone sous administration du Mouvement pour la libération du Congo (MLC) avec son quartier général à Gbadolite et une large zone d'entraves rebellions telles que le RCD/N et KML.

Human Wrigthwatch (HRW, 2003) établit que le conflit en Ituri ressurgit en juin 1999 lorsqu'un petit nombre d'Hema auraient tenté d'acheter des autorités locales afin qu'elles modifient les registres de propriété foncière en leur faveur dans la zone de Walendu Pitsi en territoire de Djugu. Ils auraient ainsi utilisé des faux papiers pour expulser les habitants Lendu de leurs terres. Les Lendu ont décidé de se venger et en l'absence d'une forte autorité locale, l'incident a rapidement viré à une confrontation entre les deux communautés.

L'ingérence de l'Ouganda, pays déjà présent dans la région à la suite de cette deuxième guerre du Congo, aggrava la situation. En effet, le Brigadier Général James Kazini, alors en charge de l'armée ougandaise en RDC, qui nomma Adèle Lotsove Mugisa, une Hema, gouverneur provisoire des districts de l'Ituri et du Haut Uélé, coïncidait avec la dispute foncière locale et donné ipso facto l'impression que l'armée ougandaise se rangeait du côté Hema.

À la fin de l'année 1999, le RCD-ML a remplacé le gouverneur Hema de la Province de l'Ituri par une personne extérieure aux deux groupes rivaux. Dans les mois qui suivirent, les combats ethniques diminuèrent pour reconnaître un nouveau regain quelques semaines après la nomination par le Colonel Muzoora d'un Hema comme chef intérimaire de la province et l'assignation en résidence surveillée du gouverneur nommé par le RCD-ML.

Le 16 mai, Wamba dia Wamba est évincé de la direction du RCD en faveur d'une figure pro-Rwanda. Sept jours plus tard, les différentes factions du RCD s'affrontèrent pour le contrôle de Kisangani. Ces circonstances contribuèrent au premier cessez-le-feu de la guerre et c'est ainsi qu'en juillet 1999, l'accord de cessez-le-feu de Lusaka fut signé par les six pays belligérants (La République démocratique du Congo, Namibie, Angola, Zimbabwe, Rwanda, et Ouganda) et, le $1^{\mathrm{er}}$ août, le MLC se joignit à l'initiative. Le RCD refusa de signer pour sa part.

Ouvert officiellement le 15 octobre 2001 à Addis-Abeba et immédiatement interrompu, le dialogue inter-congolais qui devrait régler le volet politique de l'accord de Lusaka dont le cessez-le-feu n'a pas été respecté depuis juillet 1999 reprend à Sun City, et se déroule du 25 février au 18 avril 2002, en Afrique du Sud, sous la médiation de l'ancien président botswanais Ketumile Masire.

À partir du 17 Décembre 2002, le dialogue inter-congolais connut une relance à Prétoria sous la médiation de l'ONU et de l'Afrique du Sud : l'accord de cessez-le-feu de Lusaka de juillet 1999 est complété par un accord politique global sur le partage du pouvoir pendant une période de transition de deux ans entre Joseph Kabila et quatre vice-présidents. De 1'occasion, en fin 2002, les 
troupes étrangères se sont retirées de la RD Congo. Mais dans l'Est et le Nordest, les combats impliquant une myriade de groupes armés rivaux redoublèrent d'intensité. Ils sont entretenus par le Rwanda et l'Ouganda qui entendaient garder, par des guérillas interposées, le contrôle de l'exploitation des riches ressources naturelles de la région.

En janvier 2003, ce qui fut avantageux à terme de rapprochement entre les belligérants, l'Ouganda décida d'appuyer la création du FIPI (Front pour l'Intégration et la Pacification de l'Ituri), une plate-forme initialement politique qui regroupait des factions politiques de toutes les communautés ethniques en Ituri, notamment le FNI (Front pour l'Intégration Nationale), un parti Lendu présidé par Floribert NJABU, le PUSIC (Parti pour l'Unité et Sauvegarde de l'Intégrité du Congo), le parti du chef Kahwa Mandro, le FPDC (Forces Populaires pour la Democratie au Congo), un parti dominé par des Alur et des Lugbara et dirigé par Unencan, le FRPI (Force de Résistance Patriotique d'Ituri) de Germain KATANGA qui se verra plus tard remplacé par Cobra MATATA, et, le FAPC (Forces Armées Populaires Cambodgiennes) de Jérôme KAKWAVU BUKANDE, un non originaire de l'Ituri et dont le mouvement fut hétérogène sans prédominance ethnique. Le FIPI eut comme unique objectif de déstabiliser 1'UPC (Union des Patriotes Congolais) de la ville de Bunia, car elle est devenue hostile aux intérêts de l'Ouganda et il disparut après.

\section{Convergences et Divergences des Dynamiques de deux épisodes de Violences en Ituri}

L'Ituri a connu des événements macabres comparativement à ceux de 1999 à 2004, en décembre 2017, durant des longs mois de l'année 2018 et de janvier à juillet 2019, mais avec une allure accélérée.

La Lipadhog et le Club des Amis du Droit du Congo (CAD), estiment qu'en décembre 2017, un certain vendredi 15, tout est parti d'un coin non loin du marché de Uzi (Secteur Walendu DJATSI), Groupement DIROKPA Chefferie des Bahema Nord, sur une position militaire où est érigée une barrière illégale de perception obligatoire de 200 FC par les forces armées (FARDC), lorsqu' un passant Lendu du village Tete, en Groupement Laddedjo, après avoir payé $500 \mathrm{FC}$ a revendiqué sa différence de $300 \mathrm{FC}$, mais a été menacé par le militaire qui avait perçu l'argent, lequel ordonna qu'il soit arrêté par les civils qui l'entouraient. Il s'ensuivit une altercation et le passant réussit à s'enfuir tout en étant pourchassé jusqu'au marché où il fut tabassé copieusement. Le lendemain, c'est-à-dire le 16 décembre 2017, les frères du sujet Lendu qui avait été tabassé ayant appris que leur frère a été battu chez les Hema blessèrent à la machette à leur tour trois femmes Hema du Village Maze venues dans leurs champs se situant sur une colline Lendu. En réaction, certains jeunes de la Communauté Hema venus de Maze et de Dhedja 
débarqueront dans le Village Lendu de Tete et incendieront quatre-vingt-huit cases (Lipadhoj, 2019).

La descente à Djugu de la délégation de pacification conduite par le Gouverneur de Province, alors Abdallah PeneMbaka, à Blukwa-Mbi dans le Groupement Laddedjo chez les WalenduPitsi, à Blukwa-Etat dans le Groupement Buku, chez les Bahema Nord, en date du 21 décembre 2017, eut le mérite de calmer cette nouvelle résurgence du conflit mais qui les jours d'après se verra être alimenté par toute une panoplie d'acteurs.

Jusqu'à présent, il n'y a pas eu d'enquête sérieuse mettant en lumière les causes exactes de ces incidents, et les sources des renseignements, faisant preuve de confidentialité, sont difficiles d'accès. Cependant, selon une majorité d'interlocuteurs issue de toutes les ethnies confondues, ces deux incidents ont probablement contribué de manière significative à l'accroissement des tensions qui ont mené à des divers massacres (entre 2018 et 2019).

Cela étant, il est question dans cette partie de restituer chaque évènement de ce conflit à son contexte afin de le décrypter au regard des deux grands moments historiques pour lesquels les récits restent accessibles, à savoir 1999 à 2004, et 2017 à 2019. Pour le faire, trois différents pivots nous intéressent : les acteurs, leurs motivations et les mesures de dénouement ou d'ententes.

\section{Des acteurs et stratégies}

Jadis, les événements malheureux se furent déroulés dans un environnement de crise en RDC. Alors que le pays fut éclaté en administrations rebelles, l'Ituri fut envahie par plusieurs mouvements rebelles et groupes armés notamment le MLC de Jean pierre BEMBA, le RCD-K/ML de MBUSA NYAMWISI, l'UPC de Thomas LUBANGA, le FNI de Floribert NDJABU, la FRPI de Germain KATANGA, le PUSIC de Yves KAHWA et les FAPC de Jérôme KAKWAVU et tout ce, avec le soutien de deux pays agresseurs qui sont notamment l'Ouganda et le Rwanda.

Ces groupes armés, organisés selon deux circonstances dont premièrement en forces d'auto-défense, à l'absence du pouvoir public (l'État), se sont trouvées fortement soutenus et entretenues par certaines puissances régionales à l'occurrence du Rwanda et l'Ouganda qui les formèrent et les incitèrent à se faire mutuellement la guerre, derrière laquelle se cachait le pillage des ressources naturelles de la zone embrasée.

Deuxièmement, après ce temps des guerres intenses et le bilan macabre y subséquent, les dirigeants de la plupart de ces groupes, formés sur des affinités identitaires, ont exprimé le vœu de conversion et/ou de transmutation de leurs cohortes en mouvements politico-militaires pour certains, à des partis politiques pour d'autres; d'où la nécessité de développer diverses stratégies 
en regard de ces circonstances : les premières convergent avec la lutte pour l'espace et l'affirmation du pouvoir et les secondes, y sous-jacentes, se traduisant par la quête de reconnaissance et d'alignement aussi bien qu'au sein de l'armée nationale que dans le dans le ministère de l'intérieur en qualité des partis politiques.

Aujourd'hui, ces événements se déroulent alors qu'un pouvoir public légalement établi dispose effectivement du contrôle du territoire national. D'un côté ce sont les représentants Hema qui s'estiment particulièrement visés par ces actes de cannibalisme (Lire la déclaration de la jeunesse ENTE face à la situation dans le territoire de djugu et irumu, du 28 juin 2019); de l'autre côté, les Lendu tout en confirmant que les assaillants sont de la secte CODECO et qui œuvraient dans la forêt de WAGO dont la situation géographique est connue de tous, estiment qu'il n'y a pas une guerre tribale entre Lendu et Hema; les activités insurrectionnelles de CODECO quoiqu'une milice Lendu, n'engagent aucunement la communauté Lendu (Lire la déclaration de la Société civile Djugu-nord face au récent regain et intensification d'exactions dans le territoire de djugu, Kpandroma le 22 juin 2019).

Plus loin, l'on redoutait déjà que cette recrudescence de l'insécurité dans la zone de Djugu soit la conséquence de l'hospitalité offerte par les autochtones dans la zone de BOGA, en Territoire d'Irumu.

En effet, au début de l'année 2016, des sérieux soupçons enveniment la situation sécuritaire, entre autres l'intrusion des "Banyabwisha", peuple originaire du territoire de Ruthsuru au Nord-Kivu et assimilé au Hutu du Rwanda, vers les villages Berunda et Kadilo, sous prétexte de la recherche de terres agricoles et de pâturage. Bien que cela ne soit pas une raison valable de regain des atrocités, des sources analogues reconnaissent quand même le fait que lors des leurs différents assauts, les assaillants parlaient différentes langues autre que le kilendu. Ils véhiculaient leurs messages également par le kinyarwanda et un swahili avec une accentuation rwando-ougandaise. Cette évidence dissimulerait un lien resserré entre ces émigrés Banyabwisha et les groupes rebelles d'ADF-NALU et M23. Le mode opératoire des assaillants consistant à s'attaquer à des civiles en les exécutants à base principalement de la machette, à l'incendie des villages et au pillage des bêtes, l'a démontré.

C'est ici que s'affirme la thèse d'une « rébellion en gestation », vite récupérée et répandue par le pouvoir public pour justifier des violents affrontements entre l'armée et ces factions rebelles, encore que les communautés antagonistes refusaient de croire à la résurgence d'une guerre interethnique.

En somme, bien que les migrations demeurent des caractéristiques constantes de la vie dans une région, qu'il y ait ou non un conflit, celle évoquée, cependant, ci-haut, sustentant l'inquiétude chez les autochtones, 
mérite d'être filtré pour en découvrir les insinuations. Cette note d'analyse reste luisante dans cet angle de recherche et ouvre les champs à d'autres investigations.

\section{Des enjeux et des motivations}

En 1999, lorsqu'éclatent les conflits en Ituri, des enjeux fonciers ont été évoqués comme essentiels pour provoquer le déclenchement des hostilités entre les deux communautés belligérantes sus évoquées. Ce fut une compréhension à dimension interne qui céda place rapidement à d'autres : enjeux politiques et enjeux économiques.

Économiques, parce que depuis la première guerre de libération entamée en 1996, les militaires ougandais et rwandais, avaient déjà transité par l'Ituri où ils avaient observé de près les richesses de la province à Bunia et sur les bords du Lac Albert. En novembre 1998, l'armée nationale de l'Ouganda installe une première base à Bunia. Le bois est exploité dans le centre Djugu et le Sud-Ouest précisément à Mambasa.

Après le bois exploité à Djugu et Mambasa, le déploiement militaire ougandais s'est encore accentué près des localités aurifères concentrées dans le territoire de Djugu. À Mongbwalu, dans les mines de Kilo-Moto, les soldats ougandais contrôlèrent les populations locales qui extrayaient de l'or et prélevaient une bonne partie de la production. Aussi l'Administration des Mines à Bambu, à Yalala (Centre-Est) est surveillée par les soldats ougandais.

Politiques, parce que le district de l'Ituri n'avait jusqu'en 1999 pas trop souffert de la seconde guerre de la RDC et des combats entre les armées rwandaise et ougandaise. Installée à Bunia depuis 1998, l'UPDF a aidé à attiser le feu entre les forces belligérantes et sa responsabilité alors été prouvée par certaines chancelleries occidentales qui dénonçaient sa présence comme contraire aux accords de Sun City qui prévoyaient le retrait des troupes étrangères de RDC. Sous la pression internationale, le retrait rapide de l'UPDF, qui devait être remplacée par les Casques bleus de la MONUC a créé à Bunia au début de 2003 un vide stratégique qu'ont cherché à remplir-en usage de la force - les Hema de l'UPC et les Lendu du FNI/FRPI.

. De nos jours, les doutes planent sur les enjeux et motivations des acteurs. Ni les intéressés (Hema-Lendu), ni les autorités politicoadministratives, ni les politiciens du milieu (Sénateurs, Députés Nationaux et Provinciaux), moins encore les organisations de la société civile ne les précisent. Alors une préoccupation majeure demeure : À qui profitent ces événements?

À l'approche du scrutin de décembre 2018, l'on supposait que c'était une manipulation dont le maître serait le Gouvernement de Kinshasa afin de retarder les élections prévues en décembre 2018, dans cette partie du territoire national. Pour s'en rendre compte, Corneille NANGA fustigeait déjà 
ces troubles qui selon lui étaient de nature à handicaper le calendrier électoral parce qu'à même de freiner le déploiement des équipes et matériels dans cette zone en proie à des affrontements à connotation tribale.

Par ailleurs, dans ses racines, l'on ne cesse d'évoquer un fait : le 10 juin 2017, Florent Dunji, un prêtre catholique Lendu, a été retrouvé mort dans la paroisse de Drodro. Bien que l'alcoolisme ait été la raison officielle invoquée, pour la plupart des Lendu c'était un meurtre volontaire commis par des sujets inconnus mais dans une paroisse dont le dirigeant est Hema, le Curé Nzindju. En réponse, une série de manifestations s'est organisée après la découverte de son corps. Cet incident fortuit a bel et bien ouvert le champ à d'autres jusqu'à passer à la phase de l'instrumentalisation des conflits pour des fins économiques et politiques par une diversité de tireurs de ficelles.

En effet, la guerre a des coûts indispensables qui profitent à une poignée des personnes, des cadres politico-militaires vraisemblablement. Le 02 Juillet 2019, au terme d'une visite de trois jours à Bunia, en Province de l'Ituri, le président de la RDC, Félix Tshisekedi, affirmait que les récents massacres de civils dans cette province ressemblaient à une tentative de génocide et à un complot monté pour déstabiliser le pouvoir de Kinshasa, parce qu'en fait, économiquement, le problème du financement de l'effort de guerre se pose avec une acuité nouvelle dès lors que la survie du gouvernement est en jeu.

En effet, le financement de l'effort de guerre repose certes sur les règlements opérés sur des impôts, taxes et autres droits, mais également sur l'émission de dettes publiques. Bien que cela ressorte d'une charge publique bien retracée à terme des prévisions budgétaires, les couts qu'impose la guerre affectent sérieusement l'Etat. Ceux qui en profitent vivent au sein même de cet Etat et ont généralement des pouvoirs de décisions tel qu'épluché dans les lignes précédentes.

Politiquement, la géo-localisation du bastion rebelle, dans la foret de wago, aux abords des cotes lacustres, ne cessait de faire appel à une variété d'interrogations. Comment ce mouvement subversif s'est-il recréer dans une zone pourtant sous contrôle de l'Etat, d'où tirent-il ses financements et munitions? Si l'on croit que la plaque tournante de regain des violences en Ituri vingt ans après demeure la CODECO, milice tribale proche de la communauté Lendu, l'on ne peut, cependant, s'empêcher d'authentifier - après exploitation des différents rapports rendus publics en pleine belligérance - que la CODECO avait contribué au recrutement et à l'organisation de la formation des assaillants au moins depuis le décès de Florent Dunji (voir ci-dessus) en juin 2017.

La campagne de la CODECO visait principalement les jeunes Lendu, y compris d'anciens combattants du FNI qui n'avaient pas réussi à se réinsérer dans la vie civile après les guerres de 1998-2003. L'objectif 
manifeste était au départ celui de réaffirmer une sorte d'hégémonie en vengeant les meurtres de leurs. L'on croit que derrière ce dernier se dissimulait un envahissement et une perpétuelle exploitation des richesses du pays, comme ce fut par le passé, par des troupes aussi bien internes qu'externes, les richesses naturelles, essentiellement du sous-sol, constituant le nerf de la guerre, l'inégalité d'accès aux postes de responsabilité ou aux services de base et la compétition pour le pouvoir et ces ressources auraient tout de même aidé à réanimer l'agenda des acteurs dans cette guerre.

Enfin, dans toute cette belligérance, la MONUSCO a demeuré jusqu'à ce jour, dans sa mission d'observation. Des attaques se sont déroulées sans aucune protection des civiles. Alors que jadis la présence des troupes de MONUC avait permis l'amélioration de la situation de la population civile sécurisée par les casques bleus.

Quant aux partis politiques issus des groupes armés Hema et Lendu dont l'Union des Patriotes Congolais (UPC) et le Front des Nationalistes Intégrationnistes (FNI), aucun parti n'a pris part comme jadis dans ces atrocités mais appelle les deux communautés au calme, toute en dénonçant la légèreté du pouvoir public dans la gestion de ces événements.

\section{Des ententes}

Jadis, dans les Accords de Luanda du 6 septembre 2002, l'Ouganda et la RDC avaient accepté qu'une Commission de Pacification en Ituri (CPI) soit établie pour instituer une structure intérimaire pour gouverner l'Ituri, appelée Administration Spéciale Intérimaire de l'Ituri, après le départ de l'armée ougandaise et avant qu'une administration congolaise régulière puisse être mise sur pied.

Quelques jours après le retrait de l'armée ougandaise de Bunia, les combats ont repris entre l'UPC et les milices Lendu et Ngiti, faisant des centaines de morts civils alors que chaque milice attaquait les gens du groupe opposé. La MONUC a dû augmenter l'effectif de ses soldats pour atteindre 700 mais les unités des gardes uruguayens n’étaient ni capables, ni équipées pour contrecarrer des combats de cette ampleur.

Dans la perspective d'endiguer cette flambée de violences, le Conseil de Sécurité de l'ONU a voté, le 30 mai 2003, pour la création d'une Force Multinationale Intérimaire d'Urgence pour assurer la sécurité et la protection des civils à Bunia.

Par ailleurs, le processus de paix en Ituri s'est déroulé en quatre grandes étapes. Après une première phase purement militaire visant à retirer le contrôle de Bunia à l'UPC, la MONUC, le Comité International d'Appui à la Transition (CIAT) et les autorités de transition ont lancé une initiative globale en faveur de la restauration de l'État de droit équilibrée entre dialogue, cooptation, incitation et action coercitive. 
Aujourd'hui, plusieurs efforts ont été consentis par le gouvernement provincial de la Province de l'Ituri pour atténuer la tension ethnique depuis le décès du révérend père Florent DUNDJ, de l'ethnie Lendu, de la congrégation du PÈRE CARME.

Après diverses descentes du pouvoir central dans la Province de l'Ituri, à travers les gestionnaires du secteur de l'intérieur et la sécurité nationale (RAMAZANI SHADARI et Henri MOVA SAKANI), et ATAMA TABE ; la police et l'armée furent dépêchées sur les lieux d'atrocités, une accalmie apparente s'est observée, sans identifier clairement les auteurs des événements qu'on qualifie des « assaillants ».

Après avoir constaté un redoublement des violences et insécurité, les Forces Armées de la République Démocratique du Congo (FARDC) mirent sur pieds une stratégie de guerre, baptisée "Zoruba Ya Ituri" qui mena à la récupération et au contrôle de la forêt de Wago, décrite comme bastion des assaillants. A coté de ce volet militaire de résolution de la crise, des dialogues et conférences ont été engagés.

En effet, une rencontre de pacification des filles et fils du territoire de DJUGU a été organisée par la Fédération des Entreprises du Congo (FEC) et la coordination de la Société Civile de l'Ituri à FATAKI, le vendredi 14 juillet 2017 sous la houlette de l'autorité provinciale et aurait permis à réduire tant soit peu sensiblement la nocivité des faits devenus inquiétants. De même, au terme de la conférence de Rethy/Kpandroma, sur la paix et le développement, tenue en Juillet 2019, les participants, tout en reconnaissant que des atrocités ont été perpétrées par les troupes de Ndgudjolo, exigeaient des autorités certaines mesures de décontraction : l'installation dans le territoire de Djugu du programme de désarmement, démobilisation et réinsertion ; la relève des fonctions des chefs devenus inactifs et incompétents et; l'adoption d'une nouvelle loi portant sur la gestion des terres coutumières et la concentration, l'une des causes éternelles de conflits à Djugu.

\section{Volet théorique et pistes de sortie de crise}

\section{Volet théorique du conflit}

Les facteurs explicatifs des guerres sont évidemment multiples et impliquent des approches pluridisciplinaires: politiques, sécuritaires et économiques d'une part; sociologiques et psychologiques, de l'autre. Sans vider toutes ces approches, nous présentons pour chacune des motivations qui puissent justifier leur appel dans le cadre de la présente analyse.

Sur le plan politique, l'approche géopolitique nous a poussé à développer la théorie de l'espace nourricier qui se justifie ici par le fait que les conflits survivent grâce aux soutiens extérieurs avec l'objectif de captation des ressources naturelles. 
En effet, cette note d'analyse a démontré ci-haut l'implication de certains pays voisins, principalement de l'Ouganda dans la crise en Ituri. En quête de l'espace nourricier, ce pays a aidé à armer les groupes en présence et à les exciter à se faire mutuellement la guerre afin qu'elle en profite.

Dans sa note d'analyse sur les différends frontaliers ougandocongolais, Justin Bahinduka Besisa (Bahinduka, 2019, pp 54-55) révélait que l'enjeu de confrontation entre la RDC et l'Ouganda s'explique par le fait que la rivière Semiliki contient des réserves importantes de pétrole estimées à plus de 400 millions de barils dont la découverte a été réalisée dans le versant ougandais. De ce point de vue, agrandir l'espace est une approche stratégique pour l'un ou l'autre pays. En d'autres termes, gagner du terrain revient donc à gagner les barils de pétrole. Au cœur de ces enjeux, la RDC semble quelque peu en somnolence, pour autant qu'elle a moins avancé en matière de la prospection que l'Ouganda.

Sur le plan économique, les conflits résultent d'une dialectique économique de la part de plusieurs acteurs impliqués dans la gestion de la res publica. Nous avons fait appel à deux théories, une de l'équilibre général et l'autre de contrats pour formaliser les comportements des belligérants et du gouvernement.

En effet, la guerre et la paix ont des coûts et des avantages. Partout où les hommes égaux sont rationnels, ils défendent leurs intérêts, préfèrent la paix à la guerre et ont par ce fait des droits absolus sur les biens et sur les personnes. Ceci démontre que la paix est profitable à chacun, mais la paix a surtout un coût élevé si l'autre décide de faire la guerre.

Si une autorité intervient pour faire respecter le contrat et sanctionner celui qui ne le respecte pas, il y aura un coût réduisant les dividendes de la paix, mais les solutions de paix seront préférables aux solutions de guerre.

La question que l'on veut bien se poser repose sur le poids spécifique des facteurs économiques et du sous-développement dans l'explication des conflits et dans le processus d'engrenage et de propagation non régulée qui les caractérisent.

La guerre doit être financée et peut avoir pour motivation la captation de richesses. Les conflits sont favorisés par le sous-développement économique, le chômage des jeunes, la pauvreté et l'impossibilité pour les États d'assurer les fonctions régaliennes.

Par ailleurs, selon la perspective constructiviste, le conflit identitaire repose sur une dynamique psychologique puisque les conflits identitaires semblent être le produit de blessures narcissiques à l'identité des groupes; en vérité ces blessures et la volonté de les guérir par le recours à la violence sont surtout construites par des leaders ethniques et politiques. Ces crises ne sont pas nécessairement ancestrales ou inévitables; elles correspondent à des 
déséquilibres cognitifs provoqués et alimentés par une peur chez le groupe de disparaître ou d'être diminué.

Les identités sociales à caractère agressif, dans cette perspective, sont construites socialement par le biais d'un ensemble de processus complémentaires où interviennent à nouveau les élites politiques qui se servent des vieilles mythologies politiques pour nourrir les peurs collectives qu'inspirent l'avenir (Michel, 2000, pp 55-90).

Les conflits identitaires résultent aussi du mauvais fonctionnement des quatre dimensions de la paix civile, à savoir la paix politique, la paix économique, la paix sociale et la paix culturelle, qui correspondent aux quatre espaces de citoyenneté, indispensables à la volonté de vivre ensemble. Les conflits identitaires ont une autre caractéristique, car ils tournent autour de la volonté de dominer, de commander et la quête de pouvoir de l'homme. Ici, comme dans d'autres types de conflits, l'homme veut réaliser un dessein politique qui débouche sur un pouvoir.

\section{Piste de solutions}

C'est la nature prédatrice de la gouvernance de la RDC, le gain que procure l'économie de guerre aux acteurs, le désœuvrement des jeunes, singulièrement d'anciens combattants, la culture de l'impunité qui a élu domicile en RDC, l'absence d'un plan de défense de la sécurité proactif, mais surtout l'instrumentalisation de ces conflits à des fins politiques qui justifient la persistance et la résurgence des conflits en Ituri. Pour ce faire, tout mode de résolution qui serait proposé sans tenir compte des spécificités internes à la situation conflictuelle ne saurait être efficace.

Afin de booster une cohabitation des ethnies antagonistes en Ituri, cette note d'analyse s'assoit sur deux types de propositions. Le premier politique et sécuritaire ; le second social et psychologique.

Sur le plan politique et sécuritaire, le rétablissement de l'autorité de l'État sous toutes ses formes se révèle comme pilier primordial à tout autre mécanisme d'instauration de la stabilité. Ceci passe impérativement par cinq axes prioritaires suivants:

Premièrement, avec une neutralité réaménagée, l'État devrait revenir sur les délimitations des limites de 1960 et installer une commission chargée de surveillance. L'on se rappelle qu'au courant de l'année 2007, l'Union Européenne, en collaboration avec certaines structures nationales et locales, avait proposé de mettre en œuvre une commission foncière en Ituri chargée de mener des actions de prévention et de sensibilisation sur les conflits fonciers. Installée, cette commission fit face à des problèmes énormes liés notamment au budget de son fonctionnement. Les traces de cette commission se révélant inaperçues, l'État devrait arriver à relancer cette ingéniosité tactique qui 
réinventerait un nouvel air au sein de la population habitant cette partie du pays (DJUGU).

Deuxièmement, revenir sur clauses de coopération économique consignées dans l'accord de NGURDOTO entre la RDC et l'Ouganda. En effet, il est confirmé aujourd'hui l'implication des tierces personnes, non apparentes, dans les violences armées en Ituri ; cette mainmise des acteurs tant internes et beaucoup plus externes ne laisse passer des interrogations au sujet de leurs stratégies et motivations.

Pour les externes, ce sont les mêmes que celles évoquées jadis (19992004), c'est-à-dire, obtenir par tous les moyens possibles le pillage des ressources naturelles de la RDC. Aujourd'hui, le lac Albert, en Ituri, est une frontière naturelle, dont la limitation pose problème depuis le début des prospections pétrolières. Le passage de la prospection à la production du côté ougandais émet de sérieux doutes quant à la coopération entre les deux pays et d'aucuns estiment que les vases de l'Ituri seraient déjà en train d'être vidées malignement par l'Ouganda.

Les uns et les autres, frictionnés à dresser le bilan des meurtres à la machette et des pillages de bêtes, Abdallah Pene Mbaka, alors Gouverneur de l'Ituri, réagissant sur les ondes de la Radio Okapi au cours de l'émission Dialogue entre Congolais en date du 19 septembre 2018 (Abdallah PeneMbaka sur dialogue entre congolais, émission Radio Okapi du 19 sept.2018, sur le thème : « la persistance de l'insécurité dans l'Est de la RDC »), avait qualifié cette flambée de violences d'une rébellion en gestation, à Djugu, thèse rejetée tout au début et aujourd'hui soutenue, répandue et défendue par le pouvoir aussi bien local que national. À notre niveau, nous estimons que s'il y a une rébellion, c'est avec la participation de certains fils égarés du milieu, d'où l'armée devra arriver à avoir le contrôle de l'ensemble de la forêt et d'autres agglomérations longées sur la côte nord-ouest du Lac Albert.

Troisièmement, la dépolitisation de l'armée et la mise en place par celle-ci d'une stratégie efficace de guerre. Les forces armées doivent arriver à multiplier des raids sérieux à l'image de ceux de l'opération ZARUBA YA ITURI sur les cantonnements des miliciens identifiés ; et arriver par ailleurs à la démobilisation des groupes et à leur réinsertion au sein de l'armée nationale, avec le concours du gouvernement.

Quatrièmement, l'identification claire par les services de sécurité des assassins et des tireurs de ficelles tant internes qu'externes et leur défection devant les instances compétentes. Plusieurs rapports ont cité des mains noires dans l'amplification des violences à DJUGU, mais même pas un seul n'a osé fournir des données fiables quant à l'identité des assassins et leurs bandes. Cette situation crée des flétrissures au sein d'une population qui espère à 
l'existence d'un État et d'un pouvoir public chargés de la sécurisation des personnes et de leurs biens.

Cinquièmement, étant donné que la lecture sur les conflits invite certes de s'attaquer à ses causes, même les plus délicates que soient-elles et qu'à ces causes, il convient de trouver les moyens de prévention, à l'instar de ceux de résolution, où prévenir les conflits consisterait à privilégier l'aspect négociation ou diplomatie. Et donc, la diplomatie et la négociation sont toujours préférables à la guerre, comme d'ailleurs se contentait de le répéter Churchill : " to jaw-jaw is always better than to war-war » (Charles-Philippe David, 2013, pp 313-340). La guerre débloque une société bloquée car elle amène les belligérants autour d'une table pour aboutir au consensus.

En ce sens, les stratégies de paix constituent depuis longtemps un domaine de recherche important. Cependant, le dialogue ayant démontré ses limites du fait que les communautés se cachent mutuellement des vérités, il faudrait arriver à l'organisation des conférences de paix dans la Province de l'Ituri aux cours desquelles tout congolais vivant en Ituri pourra faire sa lecture des faits en établissant la responsabilité de chacun et se fixer une ligne de conduite future car les atrocités touchent tous les habitants du milieu sans distinction.

Sur le plan social, les méthodes s'écartent entre la psychologie et le droit. Psychologiquement, l'État doit arriver à la création d'un cadre d'encadrement psychothérapeutique visant les enseignements susceptibles d'entraner des changements des mœurs belliqueuses à celles modérées. Il s'agira ici, de décourager les jeunes gens à des pratiques thaumaturges les contraignant à s'abreuver de «mayiyambau» (Eau de MBAU) et de « dawayandege » (Médicament d'oiseau) qui, d'après Alphonse Maindo, sont respectivement un cocktail à base de plusieurs plantes investi de vertus mystiques, et une sorte de breuvage également qui cette fois-ci permettrait de mener des actions «commando» dans le camp ennemi et de s'échapper sans peine au rythme des oiseaux (Maindo, 2013, pp 180-192).

Par ailleurs, il faudrait arriver à venir en aide aux sinistrés par la mise en place ou par la réactivation des tribunaux de dommages de guerre qui auront la tâche de trancher les litiges nés des différents conflits coutumiers, fonciers, etc. Étant donné que les mêmes causes entraînent les mêmes effets, les communautés doivent respecter l'article 9 de la constitution qui sacralise la souveraineté de l'État sur le sol et le sous-sol pour stopper la globalisation et la restitution de tout conflit individuel à un conflit foncier général, constituant ainsi une échappatoire pour élucider la guerre interethnique. Les causes actuelles des atrocités évoquées par la communauté Lendu à travers certains audios et déclarations dont le décès du père DUNDJI Florent, d'ailleurs élucidées par le Vicaire du Diocèse de Bunia dans la rencontre de Fataki du 
14 juillet 2017 en présence du pouvoir public, nécessitent un éclaircissement par l'État Congolais pour stopper des acharnements pour ces mêmes causes.

\section{Conclusion}

Cette note d'analyse politique a tenté de relever les analogies et les altérités entre les événements de 1999 à 2003 et ceux dupliqués entre 2017 et 2019, séparés d'un intervalle de vingt ans.

D’aucuns pensent que les causes des affrontements des ethnies Lendu et Hema dans les territoires de Djugu et Irumu en Ituri sont foncières. Nul ne comprend le mécanisme par lequel une cause avouée n'arrive à être maîtrisée par les hommes qui en supportent impuissamment les effets. Les événements actuels ont surpris par leur allure accélérée en embrasant l'Ituri dans un temps record avec des dégâts matériels et humains considérables aux vues du pouvoir public et la Mission de l'Organisation de Nations Unies au Congo (MONUSCO).

Aucune de ces deux communautés, jadis en guerre, ne croient à l'existence d'un conflit ethnique mais une situation orchestrée ailleurs pour une fin planifiée et inavouée et dont les populations en sortent victimes. Néanmoins, les villages Hema ont été vidés de ses habitants et incendiés et les attaques provenaient selon plusieurs sources des villages Lendu environnants avec l'identification de certains brebis égarés de cette communauté parmi les déstabilisateurs. Des malfrats arrêtés par le pouvoir public comptent un bon nombre de ressortissants de la communauté Lendu Cependant, certains Lendu n'adhèrent pas non plus dans cette idée déstabilisatrice de l'Ituri, malgré la prise en charge psychologique des combattants par un mouvement magicoreligieux dénommé «CODECO » d'un sujet Lendu sans identité claire.

Au terme de la présente réflexion, l'État congolais devra se reconnaître en prenant ses responsabilités en vue de la sécurisation des personnes et de leurs biens dans ce territoire qui fait partie intégrante de la RDC et par ailleurs les communautés devraient arriver à se prêcher mutuellement la paix et à quitter l'étape de mythes assis sur le complexe, légué par le pouvoir colonial belge.

\section{References:}

1. Bahinduka, B. (2009). Les différends frontaliers ougando-congolais : un nœud gordien de la coopération bilatérale ou nouvel enjeu de conflit régional?In Journal of Social Science and Humanités Research, IJIRDO, Indes, vol.4, Avril 2019

2. Bernard, L. (2014). Le Congo belge, au cœur du continent noir, $n^{\circ} 9 \mathrm{H}$, Automne-Hiver

3. Braeckman, C. (1999). L'enjeu congolais: l'Afrique centrale après Mobutu, Paris, Fayard, 428pp 
4. Charles-Philippe David (2013). Peut-on prévenir et résoudre les conflits ?, La guerre et la paix

5. Dan, F. (2014). This Land is my Land: Land Grabbing in Ituri ? in An Ansoms, Losing Your Land: Dispossession in the Great Lakes, Boydell \& Brewer Ltd,

6. Friedrich, R. (2019). EncyclopaediaUniversalis [en ligne], consulté le 13 juillet. URL : http://www.universalis.fr/encyclopedie/friedrichratzel

7. Human Rigthwatch, RDC CONGO Ituri: "couvert de sang" violence ciblée sur certaines ethnies dans le Nord-Est de la RDC, Vol. 15, No. 11 (A), Juillet 2003

8. Lpadhoj, La reprise des hostilités en Ituri en 2017-2018: l'état congolais et la cour pénale internationale mis à l'épreuve 20 ans après, Mai 2018, consulté le 11 Juillet 2019

9. Maindo Ngonga, A. (2019). La républiquette de l'Ituri en République démocratique du Congo: un Far West ougandais, Politique africaine2003/1 ( $\left.N^{\circ} 89\right)$, pages 181 à 192, mise en ligne sur sur Cairn.info le 15/11/2012, consulté le 20 Juillet.

10. Michel, F. (2000). À l'ouest rien de nouveau? Les théories sur l'avenir de la guerre au seuil $\mathrm{du} \mathrm{xx}^{\mathrm{e}}$ ? siècle?, Études internationales, vol, XXXI, $n^{\circ} 1$

11. Nicaise Kibel'bel Oka (2016). La RD Congo-Ituri. De la guerre identitaire au pillage des mines d'or de Kilo-Moto par des multinationales anglo-saxonnes, Editions-Scribe.

12. Saif, F.(2009). Inferiority and inferiority complex, 28 février.

13. Samba Kaputo (1982). Phénomène d'ethnicité et conflits ethnopolitiques en Afrique noire postcoloniale. Le cas de l'Ituri, Kinshasa, PUZ

14. Thierry Vircoulon (2005). L'Ituri ou la guerre au pluriel, dans Afrique contemporaine2005/3 $\left(\mathrm{n}^{\circ} 215\right)$

15. Touré Maliaka Sefu (2019). Conflits de pouvoir et crise de légitimité au congo-kinshasa. Regard sur les retombées du scrutin présidentiel de Novembre 2011, EUE, Brivibas, LV-1039, RigaLatvia, European Union. 